\title{
Les premiers dictionnaires médicaux en langue anglaise : glissements diachroniques du spécialisé au non spécialisé
}

Jean-Pierre Charpy

\section{OpenEdition}

Journals

Édition électronique

URL : http://journals.openedition.org/asp/2176

DOI : 10.4000/asp.2176

ISBN : 978-2-8218-0416-6

ISSN : 2108-6354

\section{Éditeur}

Groupe d'étude et de recherche en anglais de spécialité

\section{Édition imprimée}

Date de publication : 1 mars 2011

Pagination : 25-42

ISSN : 1246-8185

Référence électronique

Jean-Pierre Charpy, "Les premiers dictionnaires médicaux en langue anglaise : glissements diachroniques du spécialisé au non spécialisé », ASp [En ligne], 59 | 2011, mis en ligne le 01 mars 2014, consulté le 02 novembre 2020. URL : http://journals.openedition.org/asp/2176 ; DOI : https:// doi.org/10.4000/asp.2176

Ce document a été généré automatiquement le 2 novembre 2020.

Tous droits réservés 


\title{
Les premiers dictionnaires médicaux en langue anglaise : glissements diachroniques du spécialisé au non spécialisé
}

\author{
Jean-Pierre Charpy
}

\section{Introduction}

1. Comme Michel Van der Yeught l'a montré dans son Mémoire de synthèse pour l'obtention de l'Habilitation à diriger des recherches (2009), l'étude diachronique des langues de spécialité constitue un objet de recherche pertinent, trop souvent délaissé. En effet, la plupart des études en anglais de spécialité sont synchroniques et font la part belle au disciplinaire et au professionnel. Il semble toutefois souhaitable que les langues de spécialité en tant qu'objets d'étude soient replacées dans une perspective diachronique et que leurs origines historiques respectives, qui constituent l'une des bornes des divers domaines spécialisés, soient repérées et analysées.

Charles Bazerman avait tracé une voie majeure à la démarche diachronique en publiant Shaping Written Knowledge: The genre and activity of the experimental article in science (1988). D'autres chercheurs, comme Dwight Atkinson (1999) et Ellen Valle (1999), ont apporté un éclairage significatif sur le discours scientifique et l'article de recherche scientifique en proposant des études diachroniques à partir de The Philosophical Transactions of the Royal Society of London. Plus récemment, Alan Gross, Joseph Harmon et Michael Reidy (2002) ont étudié l'article de recherche scientifique selon une perspective diachronique plurilingue. Cela fait également plusieurs années déjà que David Banks travaille sur les origines de l'anglais scientifique et sur son évolution selon une perspective diachronique; une série d'articles publiés dans la revue ASp (2004, 2006 et 2009) témoigne de la constance et de l'intérêt de ses travaux de recherche. 
3 Néanmoins, à ce jour, les origines de l'anglais médical en tant que langue de spécialité restent plutôt obscures, même si des avancées notables sur l'évolution du langage médical ont été effectuées récemment par Françoise Salager-Meyer, Maria Àngeles Alcazar Ariza et Nahirana Zambrano d'une part (2003), et Britt-Louise Gunnarsson d'autre part (2007).

Dans cet article, qui s'inscrit dans le cadre général de la linguistique historique et dynamique, mais aussi dans le contexte particulier de l'anglais de spécialité, je propose de remonter les siècles afin d'étudier l'émergence des premiers glossaires et dictionnaires médicaux en langue anglaise. Dans un premier temps, je fais référence à l'analyse du spécialisé en tant que tel, proposée par Michel Petit (n.d.) dans une note de position publiée sur son site ${ }^{1}$. Je m'appuie ensuite sur l'étude, réalisée en 2009 par Michel Van der Yeught, du spécialisé comme objet d'observation indépendant afin de tenter de délimiter le passage de la langue spécialisée (l'anglais médical) à la langue générale (l'anglais généraliste), c'est-à-dire de repérer le moment où il apparaît nécessaire d'éclairer le sens de termes spécialisés en ayant recours à des mots non spécialisés.

5 Afin de définir l'aire spatiale, temporelle et linguistique de la transition spécialisé (S)non spécialisé (NS) permettant de poser l'existence de la langue de spécialité, je répertorie l'émergence d'outils lexicographiques comme les premiers glossaires et autres dictionnaires, outils privilégiés de l'affirmation d'une communauté linguistique professionnelle. En effet, je postule, avec M. Van der Yeught, que ces signes tangibles constituent la preuve que cette communauté doit communiquer avec l'extérieur afin d'établir un pont avec le non spécialisé.

6 L'empan diachronique de cette étude porte essentiellement sur les $17^{\mathrm{e}}$ et $18^{\mathrm{e}}$ siècles en Grande-Bretagne. Après avoir présenté le contexte lexicographique général de cet empan - en particulier, les premiers dictionnaires généralistes appelés«hard word dictionaries» - je tente de repérer l'affirmation de la communauté spécialisée dans le contexte où les premiers dictionnaires médicaux ont vu le jour.

$7 \mathrm{Au}$ début du $17^{\mathrm{e}}$ siècle, l'art empirique de la médecine médiévale prédomine et l'utilisation des plantes médicinales est extrêmement populaire. LeCollege of Physicians impose l'utilisation systématique du latin. Les premiers glossaires ou dictionnaires médicaux en anglais sont de simples traductions d'ouvrages exogènes en latin. Pour ce qui est du domaine spécialisé de l'anglais médical, mes recherches initiales montrent que A Physical Dictionary, publié en 1657 pour le compte d'un certain John Garfield, pourrait bien marquer une transition initiale S-NS. Cet ouvrage a pu permettre à un lectorat composé en partie de non-spécialistes de mieux comprendre ou de s'approprier la langue spécialisée de la communauté médicale. À la lumière des travaux d'un chercheur finlandais, Jukka Tyrrkö, je cherche à comprendre les raisons socioéconomiques et idéologiques qui ont encouragé des lexicographes et l'éditeur à rédiger ce premier glossaire-dictionnaire spécialisé et à le publier dans une période particulièrement agitée de l'histoire britannique, marquée par l'avènement des idées puritaines et de l'empirisme.

8 Après avoir repéré ce premier passage S-NS au $17^{\mathrm{e}}$ siècle, je pose la question de savoir si ce glossaire marque un passage significatif ou si d'autres dictionnaires médicaux plus tardifs comme The Physical Dictionary de Steven Blankaart (1684) ou le Lexicon PhysicoMedicum;or, a New Physical Dictionary de John Quincy (1719) constituent une confirmation plus tangible de ce passage. J'élargis ma recherche aux dictionnaires 
médicaux de type encyclopédique publiés au $18^{\mathrm{e}}$ siècle en Grande-Bretagne. C'est dans ce contexte éclairé que s'inscrit le dictionnaire du médecin anglais Robert James $A$ Medicinal Dictionary, including Physic, Surgery, Anatomy, Chymistry and Botanic, in all their Branches relative to Medicine, publié en trois volumes entre 1743 et 1745. Son dictionnaire semble représenter un jalon majeur dans le balisage du domaine spécialisé.

Je cherche également à savoir si ces dictionnaires, marqués par l'empreinte de la Raison et l'intégration des domaines spécialisés dans le champ des connaissances universelles, ont joué un rôle dans l'affirmation d'autres communautés spécialisées, en particulier aux États-Unis au $19^{e}$ siècle.

\section{De la nécessité d'étudier l'objet « langue de spécialité » selon une perspective diachronique}

10 À ce jour, mes travaux de recherche ont porté essentiellement sur un domaine spécialisé, celui de l'anglais médical, et sur ses aspects professionnels et disciplinaires dans une perspective synchronique. Si cette approche a pu donner lieu à des applications disciplinaires diverses à court terme, en particulier dans le contexte de la fiction à substrat professionnel (Charpy 2004a, 2004b, 2005 et 2010), elle présuppose l'existence du domaine spécialisé et de la langue de spécialité sans en montrer les origines.

11 Afin d'observer cet objet d'étude avec plus de rigueur scientifique, j'ai estimé utile de prendre un peu de recul et de replacer l'étude du domaine spécialisé dans une perspective diachronique, à savoir dans la durée du domaine spécialisé concerné. D.Banks a mis en évidence l'intérêt d'adopter une approche diachronique dans l'étude linguistique des domaines spécialisés et des genres afin de mieux éclairer le présent :

I have come to believe that while synchronic study of a language is obviously possible, consideration of how it has come to be the way it is enhances and informs our understanding of its functions and structures in our contemporary situation. (2009a:6)

Dans le but de poser un cadre épistémologique permettant de mieux cerner la notion de spécialisé, je me suis tourné vers quelques considérations théoriques proposées par M.Petit. Dans l'espoir de repérer le passage diachronique S-NS, marqueur tangible de la reconnaissance linguistique d'un domaine spécialisé dans le cours de l'histoire, je me suis largement inspiré des travaux de M. Van der Yeught.

\subsection{Du spécialisé : quelques définitions séminales proposées par Michel Petit}

Dans l'un des éditoriaux d'ASp, M. Petit donne une définition de l'anglais de spécialité qui permet de baliser le champ de recherche de notre communauté :

L'anglais de spécialité est la branche de l'anglistique qui traite de la langue, du discours et de la culture des communautés professionnelles et groupes sociaux spécialisés anglophones et de l'enseignement de cet objet. (2002:2-3)

14 De cette définition découle une conception du spécialisé axée autour du professionnel et du disciplinaire. Dans un article plus récent, M. Petit définit ce qu'il entend par «l'anglais de spécialité stricto sensu». 
[...] cet autre volet de l'anglais de spécialité qui ne définit pas son objet par rapport à une finalité d'enseignement mais comme simple (!) objet de connaissance en soi pour l'angliciste. (2008: 23) sur la notion de spécialisé, perçus comme objets d'étude en tant que tels. Dans une note de position accessible sur son site personnel, M.Petit a encore affiné le périmètre du champ de recherche en distinguant entre trois types de spécialisé (le professionnel, le disciplinaire et le spécialisé du troisième type). En les ancrant dans une réalité sociale, il laisse entrevoir des perspectives d'analyse diachronique prometteuses :

Dans la définition qu'on a donnée, qui vise la discipline dans son ensemble, le spécialisé n'est pas déterminé par les objectifs spécifiques de l'enseignement-ce qui supposerait qu'il n'est qu'une caractéristique artificielle (un artefact de l'enseignement)-mais correspond à une donnée fondamentale de la langue-culture, « préconstruite » par le social. Il y a du spécialisé dans la LC parce que la société est structurée par des spécialisations. (Petit n.d.)

\subsection{Spécialisé-non spécialisé : la contribution de Michel Van der Yeught}

M. Van der Yeught (2009b) propose une approche théorique originale des langues de spécialité par la spécialité, plutôt que par les dimensions disciplinaires, qui ont déjà fait l'objet de nombreuses études :

Il s'agit donc d'insister sur le fait qu'avant d'être de simples matières d'enseignement, les variétés spécialisées de l'anglais sont des objets linguistiques et des objets de recherche. (2009b: 8)

Dans un contexte historique, le spécialisé précède les manifestations disciplinaires et son émergence, liée à des facteurs externes, participe à la construction des langues de spécialité. Ainsi, les langues de spécialité, bien qu'elles soient intégrées au système des langues naturelles, n'en sont pas des extensions spontanées, mais s'en distinguent par leur spécificité.

Je pense au contraire que le spécialisé existe parce qu'il se distingue du non spécialisé qui caractérise la langue générale. C'est la langue générale qui permet aux locuteurs spécialisés de communiquer entre eux. (Van der Yeught 2009b : 23)

D.Banks met l'accent sur l'importance de bien connaître les évolutions de la langue relevant du domaine spécialisé :

What is true of the language in general is also true, I believe, of specialized language, so that the problems specific to specialized language learning may become less problematical when the way specialized language has developed is understood. (2009a: 6)

19 Selon M. Van der Yeught, le marqueur indéniable de l'ancrage d'une langue de spécialité dans la langue générale est constitué par les premiers dictionnaires ou glossaires spécialisés :

En termes imagés, la dictionnarisation coupe le cordon ombilical entre la langue générale et la langue spécialisée et constitue, mieux que toute autre déclaration, un symptôme de son émergence. Elle peut être codée et résumée ainsi :

1. extraction $\mathrm{NS}=>\mathrm{S}$ (le spécialisé est extrait du non spécialisé)

2. regroupement $\mathrm{S}$ (les éléments spécialisés sont réunis et classés)

3. glose $\mathrm{S}=>\mathrm{NS}$ (un pont signifiant est établi entre le spécialisé et le non spécialisé)

(Van der Yeught 2009b : 33) 
L'objectif principal de cette étude sera donc de définir l'empan diachronique de cette transition dans le domaine spécialisé de l'anglais médical, tout en repérant le ou les premiers pontages signifiants entre le spécialisé et le non spécialisé ainsi que les facteurs externes susceptibles de les avoir provoqués.

\section{De l'intérêt de définir un cadre diachronique pour repérer les premiers glossaires et dictionnaires spécialisés} l'antiquité gréco-latine et dont la présence est toujours attestée dans la langue spécialisée à ce jour. Toutefois, son émergence en tant qu'objet linguistique distinct est un processus lent qui a bénéficié des évolutions de la pratique et de la théorie médicales au Moyen Âge et à la Renaissance. L'empan diachronique effectif de cette étude va du $17^{\mathrm{e}}$ au $19^{\mathrm{e}}$ siècle. Toutefois, avant d'aller plus en avant, je souhaite m'arrêter sur quelques mots clés hérités des siècles précédents et associés au domaine de la médecine.

Le terme «leechcraft» n'a rien à voir avec l'utilisation des sangsues dans le cadre de la saignée préconisée par les médecins de l'époque. Il s'agit d'un terme d'origine anglosaxonne désignant l'art empirique du «laece» ${ }^{2}$. Cette forme d'empirisme a marqué la pratique médicale jusqu'à la fin du Moyen Âge et a inspiré les herbiers de la Renaissance.

Le substantif «physick» fait référence à la rationalisation de l'art de la médecine, fondé sur la connaissance de la nature («physica»). On retrouve ce terme dans le titre de nombreux traités de médecine, comme dans la traduction (en 1655) par Nicholas Culpeper de The Practice of Physick, écrit à l'origine par le médecin français Lazarus Riverius.

L'expression latine «materia medica» est une notion essentielle. Elle désignait alors l'étude des plantes médicinales, de leurs propriétés et de leur utilisation thérapeutique et elle peut être considérée comme l'ancêtre de la pharmacologie moderne.

Ces termes ont encore une influence certaine au début du $17^{\mathrm{e}}$ siècle dans le domaineS, mais avant d'aborder le champ spécialisé de la lexicographie médicale et ses évolutions au cours de l'empan diachronique que j'ai retenu, il convient de présenter le contexte plus large des premiers dictionnaires monolingues NS.

\subsection{Du non spécialisé : les premiers dictionnaires généralistes en langue anglaise}

Dans un document de travail intitulé «The lexicons of early modern English», Ian Lancashire attire l'attention sur l'émergence des premiers glossaires NS en langue anglaise :

Yet the flood of words from foreign languages into English caused many readers trouble. For them, publishers put out hard-word glossaries, including Coote's English Schoole-Maister (1596), Cawdrey's Table (1604), John Bullokar's Expositor (1616), and Henry Cockeram's Dictionarie (1623):they prefigure later full English dictionaries. (2003:3) 
glossaires, ou plus exactement ces recueils de termes étrangers ou rares, associés à leurs définitions et destinés au profane, avaient une portée restreinte car ils ne couvraient pas le lexique général de la langue anglaise. Ainsi, le premier véritable glossaire monolingue en langue anglaise, A Table Alphabeticall, compilé par le maître d'école Robert Cawdrey en 1604, propose-t-il seulement un peu plus de 2500 mots difficiles, classés par ordre alphabétique, à l'usage d'un public non érudit. Quelques années plus tard, en 1616, John Bullokar compile An English Expositor. Bien que médecin de profession, ce lexicographe s'attache à définir les mots difficiles ou rares de la langue anglaise, mais ne produit en aucun cas un glossaire spécialisé ou technique. Le premier véritable dictionnaire NS tout en anglais n'a vu le jour qu'en 1656. Il s'agit de Glossographia, compilé par un lexicographe confirmé, Thomas Blount. Ce dictionnaire, considéré comme le plus complet et le plus précis à l'époque, faisait état des sources primaires et de l'étymologie des mots peu usuels qu'il définissait.

La question est de savoir si ces premiers «hard word dictionaries» ont pu avoir une influence quelconque sur l'émergence de la lexicographie spécialisée. The Oxford History of Lexicography propose la réponse suivante :

The content of Bullokar is less clear-cut than that of Cawdrey and Cockeram. As a medical man he would be expected to have introduced numerous terms of Anatomy and Physiology as well as of herbal medicines and pathological conditions. McConchie, in Lexicography and Physicke (1997), has examined the content of all three of the above works and expressed reservations about their role as precursors of specialized technical dictionaries. In the case of Cawdrey and Cockeram, he confirms our findings that the number of technical terms is almost vanishingly small; with Bullokar he describes the situation as less clear-cut, but concludes nevertheless that even the expected complement of medical terms fails to be present. (Cowie 2009 Vol. II : 54)

C'est donc ailleurs, dans le contexte idéologique et socio-économique de la profession et du monde de l'édition, qu'il convient de chercher les facteurs ayant contribué à l'émergence des premiers glossaires ou dictionnaires médicaux destinés, dans un premier temps, à un lectorat de spécialistes ne maîtrisant pas pleinement le latin.

\subsection{Du spécialisé : les premiers glossaires ou dictionnaires médicaux d'origine exogène}

Dans le domaine spécialisé de la médecine, la fin du Moyen Âge a vu le retour des théories classiques d'Hippocrate et de Galien grâce aux traductions latines de textes d'origine grecque.

By the time medieval European healers were practising their art, medical learning was slowly recovering lost ground, classical terminology was just beginning to be reintroduced and the complexities of earlier medical theory were slowly rediscovered through mostly Latin translations of Greek texts. (Tyrkkö 2006 : 155)

31 À l'aube du $17^{\mathrm{e}}$ siècle, l'art empirique de la médecine médiévale («leechcraft») subsiste et l'utilisation thérapeutique des plantes médicinales («materia medica») est extrêmement répandue. Parallèlement à la redécouverte des classiques, le Royal College of Physicians impose l'utilisation systématique du latin. L'ouvrage de référence de la communauté médicale britannique reste longtemps le Lexicon Medicum Graeco-Latinum compilé à Bâle par Bartholomaeus Castellus en 1598. 

traductions d'ouvrages exogènes en latin. C'est le cas de The English Physitian, publié en 1652, et inspiré des herbiers de la Renaissance. Son auteur, N. Culpeper, un apothicaireastrologue, avait également traduit plusieurs traités du médecin français Lazarus Riverius.

En 1649, par le biais de la traduction anglaise de The Pharmacopoeia Londonesis du Royal College of Physicians, N.Culpeper précise ses intentions en s'en prenant directement à cette vénérable institution. Dans une publication électronique, le phytothérapeute Dylan Warren-Davis cite le traducteur iconoclaste :

I am writing for the Press a translation of the Physicians' medicine book from Latin into English so that all my fellow countrymen and apothecaries can understand what the Doctors write on their bills. Hitherto they made medicine a secret conspiracy, writing prescriptions in mysterious Latin to hide ignorance and to impress upon the patient. They want to keep their book a secret, not for everybody to know. (2005: 4-5)

De telles traductions étaient très mal considérées par les institutions médicales comme le Royal College of Physicians, qui rejetaient cette forme de vulgarisation menaçant leur pré carré. Toutefois, elles n'étaient pas prioritairement destinées aux profanes, mais aux professionnels et aux apothicaires qui ne lisaient pas le latin, ce qui ne fait pas d'elles un signe probant du passage S-NS :

It is worth noting that although he advanced the printing of medical information in English, Culpeper did not advocate the dissemination of medical knowledge to laymen, only to non-Latinate practitioners of medicine. (Tyrkkö 2009 : 181)

\section{De la pertinence du passage du spécialisé au non spécialisé}

Il convient maintenant de repérer les premiers dictionnaires spécialisés d'origine endogène dans le domaine médical, visant à la fois la communauté médicale et un public instruit de non-spécialistes. Comme l'indique M. Van der Yeught, ce repérage peut permettre de circonscrire la période de transition S-NS dans laquelle l'anglais médical spécialisé se distingue clairement de l'anglais généraliste :

Même si les LSP sont apparues dans des discours antérieurs à leur publication, les premiers dictionnaires spécialisés sont les premiers à esquisser les limites de leur périmètre terminologique grâce aux trois opérations d'extraction, de regroupement et de glose. (2009b : 35)

Si le latin reste la langue de prédilection des intellectuels et des scientifiques, la langue naturelle - l'anglais - s'impose peu à peu dans l'activité intellectuelle comme l'explique The Oxford History of Lexicography:

Until the middle of the seventeenth century [...] Latin remained the customary language not only of scholars and theologians but also of philosophers and scientists. At the end of the seventeenth century, however, this situation changed drastically: national languages gained influence everywhere, almost entirely replacing Latin in all areas of intellectual activity. (Cowie 2009 Vol. I : 65)

C'est alors que la nécessité de compiler des glossaires ou des dictionnaires accessibles aux non-spécialistes s'impose, en particulier dans le domaine spécialisé de l'anglais médical. Une réponse est donnée par The Oxford History of Lexicography: 
While certain specialist lexicons appeared as early as the seventeenth century, particularly in the well-defined subjects such as Medicine and Mathematics, it was not until the eighteenth that the gradual differentiation of Natural Philosophy into what approximates the present-day branches of science and technology led dictionaries to follow suit. (Cowie 2009 Vol. II : 49) est toutefois le résultat de forces sociales, économiques, politiques et religieuses qu'il est important de décrire avant de procéder à tout repérage.

\subsection{Du $17^{\mathrm{e}}$ siècle : le rôle des facteurs socio-économiques}

M. Van der Yeught a posé le cadre théorique dans lequel s'inscrivent les différentes publications des premiers glossaires-dictionnaires spécialisés :

Elles n'aboutissent que lorsqu'une pression socio-économique puissante pousse un nombre croissant d'acteurs sociaux à œuvrer à la finalité d'un domaine spécialisé donné. Elles apparaissent lors de périodes de développement économique accéléré, de progrès technico-scientifique rapide et de mobilité sociale. (2009b : 34)

Dans le domaine spécialisé de l'anglais médical, la combinaison de plusieurs facteurs a contribué à l'émergence d'ouvrages spécialisés d'origine endogène au $17^{\mathrm{e}}$ siècle. D'un point de vue strictement socio-économique, l'essor de l'imprimerie a facilité l'accès des profanes aux textes médicaux. Des dictionnaires médicaux rédigés en latin étaient à la disposition de la communauté spécialisée, mais la nécessité de proposer au grand public des explications dans la langue vernaculaire se faisait de plus en plus pressante. Devant les impératifs économiques des membres du Royal College of Physicians, qui imposaient l'usage du latin à l'université et la publication d'ouvrages médicaux en latin, d'autres forces, issues de la pensée puritaine et de la communauté empiriste des apothicaires et des praticiens, affirmaient leur présence et leur influence comme le souligne J. Tyrrkö :

The financial interests of the learned medicos were very much at stake, and thus, predictably, their chief rivals, the empirics, saw language as a significant part of the controversy. The empirics not only questioned the scholastic tradition and embraced new theories based on first-hand observation and experiment, but also argued that medical works should be printed in the vernacular. (Tyrrkö 2009 : 172)

41 Ce conflit d'intérêts économiques divergents est également le reflet de la lutte idéologique et politique que se livrèrent les forces royalistes conservatrices et les forces réformistes puritaines avant et pendant la période de l'histoire anglaise correspondant à ce que les historiens appellent «l'Interrègne». Entre la défaite de Charles $\mathrm{I}^{\mathrm{er}}$ en 1645 et la restauration de la monarchie en 1660 , la presse, dégagée du joug royal, put jouir d'une grande liberté. De la même manière que, dans le domaine religieux, les puritains encourageaient la publication de textes sacrés en langue vernaculaire, dans le domaine scientifique, ils contribuaient à la production d'ouvrages en anglais. Il semble d'ailleurs que plusieurs textes médicaux publiés dans le quartier de Cornhill à Londres, l'aient été par des éditeurs ou des libraires proches de la mouvance empiriste et/ou puritaine. Ainsi, Hannah Allen et son époux Livewell Chapman, qui étaient imprimeurs dans ce quartier, produisaient-ils à la fois des textes religieux d'obédience puritaine et des textes médicaux. Gertrude Dawson, une amie de H. Allen, avait publié dès 1647 The Urinal of Physick de Robert Recorde, un glossaire médical bilingue destiné aux médecins ou aux apothicaires qui ne maitrisaient ni le grec, ni le latin. 
apparaît que l'ouvrage purement endogène auquel nous allons nous intéresser maintenant ait été produit dans ce contexte socio-économique et politique.

Against this backdrop, the printing of the Physical Dictionary may be seen at least partly as a product of the upsurge the empirical and puritan movements enjoyed during the Interregnum. (Tyrrkö 2009 : 173)

\subsection{A Physical Dictionary: un passage S-NS significatif ?}

Bien que certains spécialistes de l'histoire de la médecine ${ }^{3}$ attribuent la paternité du premier dictionnaire médical britannique à de Steven Blankaart, auteur de The Physical Dictionary (1684), il convient de préciser qu'il s'agit de la traduction d'un ouvrage exogène, publié quelques années plus tôt (1679) à Amsterdam sous le titre Lexicon Medicum Graeco-Latinum. Pour ce qui est du domaine spécialisé de l'anglais médical, nos recherches initiales montrent que A Physical Dictionary, publié en 1657 pour le compte d'un certain John Garfield, un des éditeurs du quartier de Cornhill (qui avait été en contact avec L. Chapman alors qu'ils étaient apprentis chez H. Allen) pourrait être le premier dictionnaire-glossaire marquant un glissement S-NS. C'est la thèse que défend J. Tyrrkö dans un article séminal de quelque 1700 mots sur cette publication largement oubliée depuis :

The main objective of this paper is to present a lexicographical description of $A$ Physical Dictionary with a particular view to demonstrating how it was a substantial medical reference work at a time when none existed in the vernacular, as well as showing its particular propensity for apothecary terminology. (2009:171)

Cet ouvrage a fait l'objet de deux publications distinctes la même année, sous forme de glossaire, en complément de la traduction anglaise (1657) du traité médical du médecin royal français Jean de Renou A Medicinal Dispensatory, Containing the Whole Body of Physick, initialement publié à Paris en 1608, et sous forme de dictionnaire publié à compte d'éditeur. Plusieurs éléments plaident en faveur d'une première transition SNS. Il s'agit d'un ouvrage endogène destiné à une communauté spécialisée de médecins, de chirurgiens et surtout d'apothicaires, souvent mal à l'aise face au lexique médical dominant inspiré du latin. Néanmoins, l'ouvrage publié pour J. Garfield n'est pas destiné aux seuls spécialistes : il comporte des définitions claires, en anglais, de termes médicaux spécialisés, facilement compréhensibles par le public comme le montre la définition suivante du mot spécialisé «Aneurism»: «A swelling caused by the breaking the internal coat of an Artery, the external being whole» (Tyrrkö 2009: 179). En revanche, d'autres éléments vont à l'encontre d'une transition S-NS définitive. John Garfield, libraire de profession, n'avait pas de compétences médicales et le lexicographe (ou les lexicographes) qui a (ont) compilé le dictionnaire reste(nt) non identifié(s) à ce jour ${ }^{4}$.

En outre, cette compilation peut être considérée comme un glossaire-dictionnaire spécialisé qui ne présente pas un échantillonnage lexical véritablement représentatif de la médecine. Si de nombreuses entrées ont trait à la rubrique «materia medica», susceptible d'intéresser les apothicaires, le domaine de l'anatomie est négligé et le champ lexical vernaculaire des maladies est sous-représenté. Toutefois, comme le fait remarquer J. Tyrrkö, l'obstacle principal à une dictionnarisation pleinement endogène réside dans le fait que ce sont des mots latins ou grecs qui constituent, pour l'essentiel, les entrées de cet ouvrage.

The headwords, which include nouns, verbs and adjectives, are primarily Latin or Greek, either in the original form or slightly anglicized. The dominance of foreign 
lexicon is such that the Physical Dictionary could arguably be considered a Latin-

English, rather than monolingual, dictionary. (2009 : 177) dictionnarisation de la terminologie boursière en contexte anglophone, M. Van der Yeught indique que «[1]e processus de séparation de la terminologie boursière hors du contexte discursif aura nécessité près de 150 ans» (2009b: 58-59). Cette évolution progressive est également observable lorsqu'il s'agit d'étudier la transition S-NS dans le domaine de l'anglais médical, qui ne peut se limiter à la publication d'un glossairedictionnaire dont le tirage modeste n'a pas contribué à une diffusion NS très large. J'ai indiqué précédemment que le glossaire-dictionnaire médical compilé pour J. Garfield constituait un point de repère essentiel. Quelques décennies plus tard, The Physical Dictionary de S. Blankaart représente une forme de dictionnarisation plus aboutie, car linguistiquement plus étoffée que celle de son prédécesseur. En revanche, cet ouvrage reste une simple traduction d'un dictionnaire exogène initialement rédigé en latin ${ }^{5}$. Après avoir repéré cette première transition significative $\mathrm{S}-\mathrm{NS}$ au $17^{\mathrm{e}}$ siècle, je complète maintenant cette étude diachronique en observant quelques dictionnaires médicaux de type encyclopédique publiés au $18^{\mathrm{e}}$ siècle.

\subsection{Du $18^{\mathrm{e}}$ siècle : le terreau fertile du siècle des lumières}

Le $18^{\mathrm{e}}$ siècle a vu le passage progressif d'une pratique médicale classique fondée sur la théorie des humeurs d'Hippocrate, qui justifiait la saignée et la purge, à une observation clinique beaucoup plus scientifique. Le dictionnaire du médecinapothicaire J. Quincy, A New Physical Dictionary, publié en 1719 et enrichi par des termes spécialisés liés à la théorie mécanique de l'iatrophysique, a contribué à cette évolution. Cette publication qui, comme l'adjectif «New» l'indique dans son titre, voulait se démarquer du travail lexicographique de S.Blankaart, n'en reste pas moins l'adaptation d'un ouvrage exogène de la fin du $16^{e}$ siècle, le Lexicon Medicum GraecoLatinum de B. Castellus. D'autres ouvrages, dictionnaires portatifs de santé ou autres traités sur la médecine domestique, contribuèrent au développement de la médecine populaire lors de la seconde moitié du siècle. Ce fut le cas de Domestic Medicine; or the Family Physician, publié en 1769 à l'initiative du docteur William Buchan, dont l'objectif principal était d'améliorer l'hygiène publique au travers de mesures préventives et de conseils de base pour les particuliers n'ayant pas la possibilité de s'adresser à un médecin.

Outre cette tendance vulgarisatrice, il faut garder à l'esprit que le $18^{\mathrm{e}}$ siècle a été celui des lumières, marqué par l'empreinte de la Raison. La volonté générale d'éclairer le 
peuple a favorisé la publication de dictionnaires NS de type encyclopédique et le domaine spécialisé n’a pas échappé à cette évolution.

\subsection{A Medical Dictionary de Robert James : un passage avéré ?} du médecin anglais R. James A Medicinal Dictionary, including Physic, Surgery, Anatomy, Chymistry and Botanic, in all their Branches relative to Medicine, publié en trois volumes entre 1743 et 1745 . Cet ouvrage de type encyclopédique est d'autant plus intéressant que l'ami de R. James, Samuel Johnson, auteur emblématique du plus grand dictionnaire NS du siècle des lumières en Grande-Bretagne, y a apporté sa contribution en rédigeant la dédicace et quelques articles. Certains historiens de la médecine insistent sur cette approche plus éclairée et sur le désir de diffusion vers les nonspécialistes de connaissances fondées sur une langue spécialisée désormais accessible à tous. Le dictionnaire encyclopédique de $\mathrm{R}$. James y est présenté comme référence de la communauté médicale éclairée et précurseur de la vulgarisation médicale. Parallèlement aux productions lexicographiques de J. Quincy, cet ouvrage exerce une influence significative sur la compilation des premiers dictionnaires dans le domaine spécialisé de l'anglais médical aux États-Unis.

France, Denis Diderot, Marc-Antoine Eidous et François-Vincent Toussaint on assuré la traduction du dictionnaire de R. James, devenu le Dictionnaire universel de médecine (1746-1748). Ce travail de jeunesse a inspiré Diderot lorsque, quelques années plus tard, il s'est penché sur la partie scientifique de la compilation de l'Encyclopédie.

-on dire alors que le dictionnaire spécialisé du docteur James marque le passage avéré S-NS que nous nous efforçons de repérer? Certes, il s'agit du premier ouvrage médical de référence illustré de type encyclopédique comportant environ mille pages. Son auteur principal, le médecin-lexicographe R. James, est issu de la communauté médicale anglaise. D'un point de vue linguistique et médical, ce dictionnaire fait l'objet d'une reconnaissance quasi unanime. Comme le précise la chercheuse Lulu Stine, le dictionnaire de James préconise une approche à la fois empirique et rationnelle de la médecine :

A part of James's information, however, is gleaned from his own experience and an examination of material of this kind throws light upon the experimental methods of the day. In discussing various schools of thought, the Dogmatists, the Empiricists, and the Methodists, James argues for the value of experience guided by reason. (1941: 190)

D'autres historiens de la médecine insistent sur cette approche plus éclairée et sur le désir de diffusion vers la communauté médicale et la population de connaissances utiles généralement scientifiquement fondées. Le commentaire du chercheur John Wiltshire va dans ce sens : «Robert James's Medicinal Dictionary started out, at least, as an earlier attempt at a similar diffusion of useful knowledge.»(1991: 91). S'il est vrai que ce dictionnaire a servi de référence dans le monde médical jusqu'au début du $19^{\mathrm{e}}$ siècle, l'importance disproportionnée qu'il accorde aux plantes médicinales semble indiquer que cet ouvrage est encore marqué par la médecine d'inspiration populaire. Un siècle et demi plus tard, Mark Twain dénonce le caractère désuet de certaines recommandations du dictionnaire de James dans un article critique intitulé «A Majestic Literary Fossil» publié en 1890 dans Harper's Magazine ${ }^{6}$. 

doute sur la portée réelle du dictionnaire de R. James dans le processus de transition SNS à une époque ou les encyclopédistes tentent d'éclairer le monde : «James's Medicinal Dictionary is the first of its kind with truly encyclopedic pretensions» (2010 Vol. II : 80). Le passage S-NS est consolidé et confirmé par R. W. McConchie dans son commentaire sur la préface d'un autre dictionnaire réputé, A New Medical Dictionary; or, a General Repository of Physic compilé en 1775 par George Motherby, l'héritier en ligne directe de R. James?

He also addresses his work to non-professionals. It is to be hoped that this Work will be found of general benefit to those whose circumstances will not permit of an academic education, and who are nevertheless desirous of obtaining a competent share of medical knowledge [Pref. v-vi]. (Cowie 2009 : 125)

55 À ce sujet, il est intéressant de noter que la toute première définition du terme spécialisé « placebo » apparaît dans la seconde édition du dictionnaire de G. Motherby (1785) sous une forme concise et explicite («a commonplace method or medicine») susceptible de faciliter la compréhension des non-spécialistes du domaine.

\section{Conclusion}

Le moment est venu de proposer quelques éléments de conclusion concernant la transition S-NS dans cette étude diachronique des premiers glossaires et dictionnaires médicaux en langue anglaise. Tout indique que cette transition ne s'est pas concrétisée de façon brutale, mais qu'elle a fait l'objet de glissements progressifs, tant dans le contexte linguistique que dans le cadre socio-économique et idéologique des $17^{\mathrm{e}}$ et $18^{\mathrm{e}}$ siècles. À ce sujet, The Oxford History of Lexicography fournit une indication précieuse :

English dictionaries of Medicine, like those of other traditional subjects, can trace back their beginnings beyond the sixteenth century, but come into their own only in the eighteenth, following the gradual professionalization of the readership, a process broadly speaking paralleled by the lexical transition from 'physick' to 'medicine'. (Cowie 2009 Vol. II : 77)

D'un point de vue linguistique, le premier point de repère significatif a été la publication du glossaire-dictionnaire de J. Garfield, A Physical Dictionary, dans la seconde moitié du $17^{\mathrm{e}}$ siècle. Des facteurs externes étroitement liés à la communauté empiriste de l'époque et à l'influence de la pensée puritaine ont fortement contribué à l'émergence de ce qu'il convient d'appeler, en termes diachroniques, le premier glossaire-dictionnaire médical en langue anglaise. Toutefois, d'autres glissements marquants ont eu lieu au $18^{\mathrm{e}}$ siècle. Alors que, dans le domaine de la langue générale, The Dictionary of the English Language compilé par S.Johnson, a marqué l'avènement du siècle des lumières, dans le domaine spécialisé de la médecine, le dictionnaire encyclopédique de R. James, A Medicinal Dictionary et A New Medical Dictionary; or, a General Repository of Physic, compilé par George Motherby en 1775, a confirmé le pontage entre le domaine spécialisé et le domaine non spécialisé.

58

En effet, ces ouvrages de type encyclopédique ont contribué à la fois linguistiquement, professionnellement et culturellement à la reconnaissance et à la spécificité d'une communauté médicale éclairée en Grande-Bretagne au $18^{\mathrm{e}}$ siècle, mais aussi à la vulgarisation de l'anglais médical. En outre, ils ont participé à l'affirmation de communautés professionnelles médicales dans d'autres pays anglophones comme les 
États-Unis en inspirant les premiers dictionnaires médicaux publiés dans ce pays. De nombreux médecins d'origine britannique, comme Robert Hooper et James Copland, ont fait carrière aux États-Unis et ont publié des dictionnaires reconnus par la communauté médicale. Ils ont contribué au développement de l'éducation médicale des Américains. Le plus populaire d'entre eux, A New Dictionary of Medical Science and Literature, publié en 1833, fut compilé par le docteur Robley Dunglison, qui avait été formé au sein de la prestigieuse faculté de médecine d'Édimbourg et qui eut l'honneur de faire office de médecin personnel auprès de quatre présidents américains.

Toutefois, d'autres facteurs, qui ont pu jouer un rôle non négligeable dans la transition S-NS, mériteraient d'être étudiés avec la plus grande attention. Il s'agit d'œuvres littéraires qui relèvent $a$ priori du domaine NS, mais qui ont pu néanmoins contribuer à une meilleure connaissance du domaine $\mathrm{S}$ par le lecteur profane. Ainsi, sachant que le romancier Tobias Smollett a exercé la profession de médecin parallèlement à ses activités littéraires, il serait utile de vérifier si un roman satirique comme The Expedition of Humphry Clinker, publié en 1771, ne comporte pas un contenu spécialisé lui permettant de contribuer également à ce pontage S-NS.

\section{BIBLIOGRAPHIE}

\section{Références du corpus}

Blankaart [Blankaert, Blanchard], S. 1684. The Physical Dictionary. Londres : Crouch.

Blount, T. 1656. Glossographia. Londres : T. B. of the Inner Temple.

Buchan, W. 1769. Domestic Medicine; or the Family Physician. Édimbourg : Balfour, Auld, and Smellie. Bullokar, J. 1616. An English Expositor. Londres : Legatt.

Castellus, B. 1598. Lexicon Medicum Graeco-Latinum. Rotterdam : Sumptibus Arnoldi Leers.

Cawdrey, R. 1604. A Table Alphabeticall. Londres : I.R. for E. Weaver.

Culpeper, $\mathrm{N}$ (trans.). 1649. Pharmacopoeia Londinensis. A physicall directory; or, a translation of the London dispensatory, made by the Colledge of physicians in London. Londres : Cole.

Culpeper, N. 1652. The English Physitian. Londres : Cole.

De Renou, J. 1657. A Medicinal Dispensatory, Containing the Whole Body of Physick. Londres : Cotrell and Streater.

Diderot, D. et al. 1746-1748. Dictionnaire universel de médecine. Paris : Braisson.

Dunglison, R. 1833. A New Dictionary of Medical Science and Literature. Boston : Bowen.

[Garfield, J.?] 1657. A Physical Dictionary; or, an Interpretation of such Crabbed Words and Terms of Art, as are Derived from the Greek or Latin, and Used in Physic, Anatomy, Chirurgery, and Chymistry. Londres : Imprimé par G. Dawson pour J. Garfield.

James, R. 1743-1745. A Medicinal Dictionary. Londres : Osborne. 
Johnson, S. 1755. A Dictionary of the English Language: in which the Words are Deduced from their Originals, and Illustrated in their different Significations by Examples from the Best Writer. (Two volumes). Londres : Strahan.

Motherby, G. 1775. A New Medical Dictionary; or, a General Repository of Physic. Londres : Johnson. Quincy, J. 1719. Lexicon Physico-Medicum; or, A New Physical Dictionary.Londres : Bell.

Recorde, R. 1665 [1547]. The Urinal of Physick. Londres : G. Dawson.

Riverius, L. 1655. A Physical Dictionary [Appended to The Practice of Physick (trans. Culpeper, N. et al.)]. Londres : Cole.

Smollett, T. 1967 [1771]. The Expedition of Humphry Clinker. Harmondsworth : Penguin Books.

\section{Références bibliographiques}

Anton, Sebastian. 1999. A Dictionary of the History of Medicine. Lancaster : Parthenon Publishing Group.

Atkinson, Dwight. 1999. Scientific Discourse in Sociohistorical Context: The Philosophical Transactions of the Royal Society of London, 1675-1975. Mahwah, NJ : Lawrence Erlbaum Associates.

Banks, David. 2004. « Philosophy, Science, Ideology and the establishment of science as a profession ». ASp 43-44, 69-80.

Banks, David. 2006. « The simple present and the simple past in the scientific journal article ». ASp 49-50, 119-135.

Banks, David. 2009a. « Starting science in the vernacular. Notes on some early issues of the Philosophical Transactions and the Journal des Sçavans, 1665-1700 ». ASp 55, 5-22.

Banks, David. 2009b. «Creating a specialized discourse: the case of the Philosophical Transactions ». ASp 56, 29-44.

Bazerman, Charles. 1988. Shaping Written Knowledge: The genre and activity of the experimental article in science. Madison, WI : University of Wisconsin Press.

Charpy, Jean-Pierre. 2004a. « Le roman à suspense à dominante médicale : typologie d'un sousgroupe générique ». InMémet, M. et M. Petit (dir.), Aspects de la fiction à substrat professionnel. Collection travaux EA 2025. Bordeaux : GERAS Éditeur, 65-82.

Charpy, Jean-Pierre. 2004b. « Milieux professionnels et FASP médicale : de l'autre côté du miroir ». ASp 45-46, 61-79.

Charpy, Jean-Pierre. 2005. « La FASP médicale et ses marges : textes de référence, prototextes et textes périphériques ». ASp 47-48, 83-101.

Charpy, Jean-Pierre. 2010. «FASP médicale et substrat professionnel : le miroir éclaté ». ASp 57, 49-65.

Cowie, A. P. (dir.). 2009. The Oxford History of English Lexicography (vol. 1 \& 2). Oxford : Oxford University Press.

Gross, Alan, Joseph Harmon and Michael Reidy. 2002. Communicating Science: The scientific article from the $17^{\text {th }}$ century to the present. New York : Oxford University Press.

Gunnarsson, Britt Louise. 2005. « Medical Discourse: Sociohistorical construction ». In

Encyclopedia of Language and Linguistics ( $2^{\mathrm{e}}$ éd.) vol 7. Amsterdam : Elsevier, 709-716.

Lancashire, Ian. 2003. « The Lexicons of Early Modern English ». University of Toronto. Consulté le 4 août 2010. <http://projects.chass.utoronto.ca/chwp/CHC2003/ Lancashire2.htm>. 
McConchie, R. W. 2009. " Propagating what the Ancients taught and the Moderns improved: The sources of George Motherby's A New Medical Dictionary; or, a General Repository of Physic (1775) ». InMcConchie, R. W. et al. (dir.), Selected Proceedings of the 2008 Symposium on New Approaches in English Historical Lexis (HEL-LEX) Sommerville, MA: Cascadilla Proceedings Project, 123-133.

Petit, Michel. 1999. « La fiction à substrat professionnel : une autre voie d'accès à l'anglais de spécialité ». ASp 23-26, 57-81.

Petit, Michel. 2002. Éditorial. ASp 35-36, 1-3.

Petit, Michel. (n.d.). « Note de position : la notion et les types de spécialisé ». Consulté le 4 août 2010. <http://www.langues-vivantes.u-bordeaux2.fr/frsa/pagesperso/michelpetit/ pdf/Def AngSpeNotePosition.pdf>.

Petit, Michel. 2008. « Once more unto the breach, dear friends ». ASp numéro spécial - Les trente ans du GERAS, 21-24.

Salager-Meyer, Françoise, M. A. Alcaraz Ariza et N. Zambrano. « The scimitar, the dagger and the glove: intercultural differences in the rhetoric of criticism in Spanish, French and English medical discourse (1930-1995) ». English for Specific Purposes 22/3, 223-247.

Stine, L. 1941. « Dr. Robert James, 1705-1776». Bulletin of the Medical Library Association 29. Boston : Harvard University, 187-198.

Tyrkkö, J. 2006. « Tokens, signs, and symptoms: Signifier terms in medical texts from 1375 to 1725 ». InMcConchie, R. W. et al. (dir.), Selected Proceedings of the 2008 Symposium on New Approaches in English Historical Lexis (HEL-LEX) Sommerville, MA: Cascadilla Proceedings Project, 155-165.

Tyrkkö, J. 2009. « A Physical Dictionary (1657): The First English medical dictionary ». InMcConchie, R. W. et al. (dir.), Selected Proceedings of the 2008 Symposium on New Approaches in English Historical Lexis (HEL-LEX) Sommerville, MA: Cascadilla Proceedings Project, 171-187.

Valle, Ellen. 1999. A Collective Intelligence: The life sciences in the Royal Society as a scientific discourse community, 1665-1965. Turku, Finlande : AnglicanaTurkuensia.

Van der Yeught, Michel. 2009a. «Les premiers essais de spécialisation des langues : les cas de l'anglais de l'économie, de la médecine, du droit et de la bourse ". Communication au $49^{\mathrm{e}}$ congrès de la SAES, atelier Anglais de spécialité - GERAS, à Bordeaux, 10 mai 2009.

Van der Yeught, Michel. 2009b. «Structurer l'anglais de la bourse : une approche des langues de spécialité par la spécialité ». Mémoire de synthèse présenté pour l'Habilitation à diriger des recherches. Bordeaux : Université Victor-Segalen Bordeaux 2.

Warren-Davis, Dylan. 2005. « Nicholas Culpeper, herbalist of the people ». Skyscript. Consulté le 4 août 2010. <http://www.skyscript.co.uk/culpeper.html>.

Wiltshire, John. 1991. Samuel Johnson in the Medical World: The Doctor and the Patient. Cambridge : Cambridge University Press.

\section{NOTES}

1. <http://www.langues-vivantes.u-bordeaux2.fr/frsa/pagesperso/michelpetit/defangspe.html>

2. Médecin-guérisseur dans la culture anglo-saxonne

3. C'est le cas, entre autres, de Sebastian Anton: "The first dictionary of medicine in Britain was published by Stephen Blanchard (1625-1703) in $1684 »(1999: 251)$. 
4. Si le glossaire-dictionnaire a bien été compilé par J. Garfield, il précise qu'il a fait appel à des spécialistes, à ce jour restés anonymes, pour expliquer les mots les plus technique : «[he] caused the [difficult words in Tomlinson's translation of de Renou's Dispensatory] to be explained by able persons, well acquainted with the Practice of Physicke.» (Tyrkkö 2009:176)

5. Voir supra 3.2.

6. Voir L. Stine : " 'If it had been sent against the Pretender's troops,' says Mark Twain, 'there would not have been a single survivor'. »(1941:187)

7. L'adjectif «New» fait écho au dictionnaire de R. James et lui rend un hommage indirect.

\section{RÉSUMÉS}

Dans le champ des langues de spécialité, l'étude diachronique des dictionnaires médicaux en langue anglaise à dominante médicale est un terrain de recherche qui n'a pas encore été clairement balisé. Cette étude a pour objectifs de repérer et d'analyser l'émergence des premiers glossaires et dictionnaires médicaux en Grande-Bretagne afin de jalonner le domaine spécialisé de l'anglais médical. Les définitions du spécialisé proposées par Michel Petit et l'analyse du passage du spécialisé au non spécialisé faite par Michel Van der Yeught servent de cadre théorique à cette étude, dont l'empan diachronique recouvre les $17^{\mathrm{e}}$ et $18^{\mathrm{e}}$ siècles. Le corpus prend en compte les dictionnaires généralistes et les premières compilations spécialisées d'origine exogène et endogène. Ces recherches montrent que A Physical Dictionary, publié en 1652, marque le premier passage du spécialisé au non spécialisé dans le domaine spécialisé de l'anglais médical. Au siècle des lumières, A Medicinal Dictionary, compilé par le médecin anglais Robert James entre 1743 et 1745 , confirme ce passage et annonce la publication des premiers dictionnaires médicaux aux États-Unis.

In the field of languages for specific purposes, the diachronic study of medical dictionaries published in English is a territory that remains largely unchartered. The aim of this study is to pinpoint and analyse the emergence of the first medical glossaries and dictionaries in Great Britain so as to mark out the specialised territory of medical English. The specialised domain, as defined by Michel Petit, and the analysis of the passage from the specialised to the nonspecialised domain put forward by Michel Van der Yeught provide the theoretical background to the study, which spans the 17th and 18th centuries. The corpus comprises both general English dictionaries and the first specialised compilations whether they be of exogenous or endogenous origin. This research shows that A Physical Dictionary, published in 1652, is the first instance of a transition from the specialised to the non-specialised in the specialised territory of medical English. In the Age of Enlightenment, A Medicinal Dictionary, compiled by the English doctor Robert James between 1743 and 1745, confirms this passage, and heralds the publication of the first medical dictionaries in the United States.

\section{INDEX}

Keywords : diachrony, lexicography, medical dictionary, non-specialised, specialised

Mots-clés : diachronie, dictionnaire médical, lexicographie, non spécialisé, spécialisé 


\section{AUTEUR}

\section{JEAN-PIERRE CHARPY}

Jean-Pierre Charpy est maître de conférences à la Faculté de Médecine de Dijon. Il est le coauteur du Guide pratique (Ellipses 2002) et du Cd-Rom (Edulang 2003) La communication orale scientifique en anglais, de L'anglais médical à la faculté, nouvelle édition (Ellipses 2008) et de L'article de recherche scientifique en anglais (Ellipses 2010). Ses domaines de recherche portent sur l'étude diachronique des dictionnaires médicaux, la FASP médicale et le discours scientifique. jeanpierre.charpy@u-bourgogne.fr 